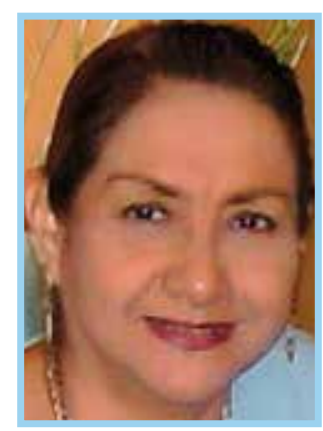

Aymé Gabriela Buitrón Aranda

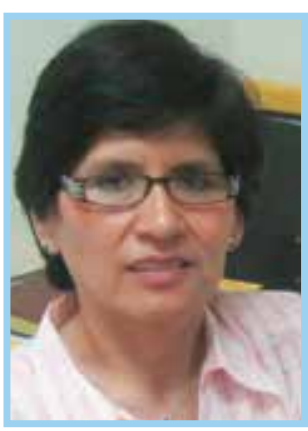

LuzCarbajal Arroyo

\section{ESTILO DE VIDA Y}

PERCEPCIÓN SOCIAL

DE ESTUDIANTES

UNIVERSITARIOS

SEGÚN GÉNERO

\section{RESUMEN}

El presente trabajo tiene como finalidad explicar la percepción de la realidad social y de la vida personal de un sector de jóvenes universitarios en el Perú, con énfasis en la diferencia de género; este artículo partirá del análisis realizado este año, en base a los datos recogidos el 2012 entre los estudiantes de la Universidad Peruana Cayetano Heredia.

\section{PALABRAS CLAVE}

Universidad, género, realidad social, vida personal.

\section{ABSTRACT}

This study has as purpose to explain the social reality and personal life perception of an area of young university students in Peru, emphasizing genre difference; this article will have basis on the analysis performed this year, according to the data collected in 2012 between students of Universidad Peruana Cayetano Heredia.

\section{KEYWORDS}

University, genre, social reality, personal life.
E xplicaremos la percepción de la realidad social y de la vida personal de un sector de jóvenes universitarios en el Perú con énfasis en la diferencia de género, este artículo partirá del análisis realizado este año, de los datos recogidos el 2012 entre los universitarios de la Universidad Peruana Cayetano Heredia. Este centro de estudios se fundó en 1962, actualmente cuenta con ocho Facultades, pero nació solo con la Facultad de Medicina y la Facultad de Ciencias y Filosofía. La universidad tiene aproximadamente 3234 alumnos. La población encuestada fue más del 50\% del sexo femenino (eran alumnos del segundo y cuarto año de estudios) representando de esa manera la mayoría femenina estudiantil en la universidad en mención.

Nosotros encontramos que los encuestados se auto identifican con la clase media y media alta en segundo lugar; opinión que se refuerza con el hecho de que $67 \%$ vive en distritos de clase media y alta, 21,9\% en distritos muy heterogéneos y sólo $11,5 \%$ reside en distritos con población mayoritaria de sectores pobres. Los distritos de clase media y alta a los que hacemos referencia son: Pueblo Libre, Lince, Jesús María, Magdalena, San Miguel, Miraflores, San Isidro, La Molina, San Borja y Surco (Ayme Buitron y Luz Carbajal, 2010). 
En el último estudio que realizamos en la Universidad Peruana Cayetano Heredia (Ayme Buitron y Luz Carbajal, 2013), encontramos que el $71,3 \%$ de los padres tiene estudios universitarios, así como el 64,2\% de sus madres, el $80,9 \%$ de sus padres tiene trabajo fijo y el $58,8 \%$ de sus madres también. El $72,5 \%$ de los padres de los encuestados tiene categoría profesional universitaria de grado superior y lo mismo el 58,8\% de sus madres. En conclusión, los estudiantes pertenecen sobre todo a una condición social media o alta, dada la condición económica estable de sus padres y el alto nivel de educación de los mismos. En comparación con el promedio económico y educativo nacional, forman parte de una élite intelectual y económica en el Perú.

Sobre la igualdad de oportunidades en el Perú, las mujeres muestran más descontento que los varones, pero no existen diferencias significativas a nivel estadístico, así los varones señalan que esta es regular y según las mujeres que es poco satisfactoria.

Tabla 1

Percepción sobre la igualdad de oportunidades

\begin{tabular}{|c|c|c|c|c|c|}
\hline & \multicolumn{2}{|c|}{ Hombre } & \multicolumn{2}{c|}{ Mujer } & Total \\
\hline & $\mathrm{N}^{\circ}$ & $\%$ & $\mathrm{~N}^{\circ}$ & $\%$ & \\
\hline Nada satisfecho & 19 & 12.9 & 35 & 12.7 & 54 \\
\hline Poco & 57 & 38.8 & 114 & 41.3 & 171 \\
\hline Regular & 60 & 40.8 & 108 & 39.1 & 168 \\
\hline Bastante & 9 & 6.1 & 19 & 6.9 & 28 \\
\hline Muy satisfecho & 2 & 1.4 & 0 & 0.0 & 2 \\
\hline Total & 147 & 100.0 & 276 & 100.0 & 423 \\
\hline
\end{tabular}

Incluso sobre la situación económica del Perú las mujeres tienen una posición más crítica que los varones, existiendo diferencias significativas a nivel estadístico entre varones y mujeres, ya que los varones están más satisfechos que las mujeres, aunque las mujeres tienen una perspectiva más optimista sobre el futuro del país.

Tabla 2

Grado de satisfacción con la situación actual a nivel económico del Perú

\begin{tabular}{|l|c|c|c|c|c|}
\hline & \multicolumn{2}{|c|}{ Hombre } & \multicolumn{2}{c|}{ Mujer } & Total \\
\hline & $\mathrm{N}^{\circ}$ & $\%$ & $\mathrm{~N}^{\circ}$ & $\%$ & \\
\hline Nada satisfecho & 3 & 2.0 & 8 & 2.9 & 11 \\
\hline Poco & 16 & 10.9 & 35 & 12.6 & 51 \\
\hline Regular & 69 & 46.9 & 178 & 64.3 & 247 \\
\hline Bastante & 56 & 38.1 & 53 & 19.1 & 109 \\
\hline Muy satisfecho & 3 & 2.0 & 3 & 1.1 & 6 \\
\hline Total & 147 & 100.0 & 277 & 100.0 & 424 \\
\hline
\end{tabular}


Tabla 3

Grado de satisfacción con la evolución del desarrollo económico del Perú

\begin{tabular}{|l|c|c|c|c|c|}
\hline & \multicolumn{2}{|c|}{ Hombre } & \multicolumn{2}{c|}{ Mujer } & Total \\
\hline & $\mathrm{N}^{\circ}$ & $\%$ & $\mathrm{~N}^{\circ}$ & $\%$ & \\
\hline Nada satisfecho & 0 & 0.0 & 5 & 1.8 & 5 \\
\hline Poco & 6 & 4.1 & 17 & 6.1 & 23 \\
\hline Regular & 49 & 33.3 & 66 & 23.8 & 115 \\
\hline Bastante & 64 & 43.5 & 157 & 56.7 & 221 \\
\hline Muy satisfecho & 28 & 19.0 & 32 & 11.6 & 60 \\
\hline Total & 147 & 100.0 & 277 & 100.0 & 424 \\
\hline
\end{tabular}

El respeto por las personas que piensan diferente según los varones es poca y según las mujeres es regular, porque los varones consideran que hay poca tolerancia entre las personas y las mujeres consideran que la tolerancia es regular, existiendo diferencias significativas a nivel estadístico entre los sexos, lo mismo sucede respecto a la percepción sobre la educación cívica de la población, así las mujeres consideran que es regular y en cambio los varones consideran que es poco satisfactoria.
Esto se puede interpretar cómo que los varones exigen a las demás personas mayor tolerancia, en cambio las mujeres exigen menos al respecto, mostrando mayor flexibilidad al entorno social. Aquí es importante añadir que las diferencias entre hombres y mujeres en función de la mayor conciencia o responsabilidad social de la mujer o cooperativismo, en comparación al varón, es resaltada por diversos estudios (G. Pérez Tarango y T. García Campos, 2010).

Tabla 4

Percepción sobre el respeto por las personas

\begin{tabular}{|c|c|c|c|c|c|}
\hline & \multicolumn{2}{|c|}{ Hombre } & \multicolumn{2}{c|}{ Mujer } & Total \\
\hline & $\mathrm{N}^{\circ}$ & $\%$ & $\mathrm{~N}^{\circ}$ & $\%$ & \\
\hline Nada satisfecho & 26 & 17.7 & 40 & 14.4 & 66 \\
\hline Poco & 58 & 39.5 & 102 & 36.8 & 160 \\
\hline Regular & 47 & 32.0 & 121 & 43.7 & 168 \\
\hline Bastante & 13 & 8.8 & 13 & 4.7 & 26 \\
\hline Muy satisfecho & 3 & 2.0 & 1 & 0.4 & 4 \\
\hline Total & 147 & 100.0 & 277 & 100.0 & 424 \\
\hline
\end{tabular}


Tabla 5

Percepción sobre la educación cívica de la población

\begin{tabular}{|l|c|c|c|c|c|}
\hline & \multicolumn{2}{|c|}{ Hombre } & \multicolumn{2}{c|}{ Mujer } & Total \\
\hline & $\mathrm{N}^{\circ}$ & $\%$ & $\mathrm{~N}^{\circ}$ & $\%$ & \\
\hline Nada satisfecho & 35 & 23.8 & 60 & 21.7 & 95 \\
\hline Poco & 53 & 36.1 & 93 & 33.6 & 146 \\
\hline Regular & 47 & 32.0 & 112 & 40.4 & 159 \\
\hline Bastante & 9 & 6.1 & 12 & 4.3 & 21 \\
\hline Muy satisfecho & 3 & 2.0 & 0 & 0.0 & 3 \\
\hline Total & 147 & 100.0 & 277 & 100.0 & 424 \\
\hline
\end{tabular}

Los jóvenes universitarios crean y recrean cotidianamente un "cosmos social personal diferenciado" esto conlleva un conjunto de prácticas, hábitos, valores, actitudes, tendencias, consumos, formas vitales. Es decir, lo que en ciencias sociales se viene denominando como Estilo de Vida.

La cultura "eficientista" surge en la década de los noventa, época en la que se destaca la primacía del mercado y que se plasmó en el proyecto tecnocrático-autoritario de Alberto Fujimori, que valoraba la iniciativa privada como eje del desarrollo (Guillermo Nugent, 2003). Es probable que el marcado interés por los estudios de los jóvenes de nuestro estudio se vea alimentado por este "eficientismo" de los noventa, años en que sus padres crecieron y alimentaron su proyecto de vida y desarrollo económico. Dicha cultura "eficientista" se nutre de una ideología económica que insiste en el individualismo y en un estilo de vida que solo puede sostenerse sobre la base del consumo. Habría que considerar que la población universitaria de nuestro estudio no evidencia un afán de ostentación con vestimentas de marca.

Al respecto describiremos algunas de sus actividades recreativas, en las que vemos que se realizan en su hogar o en el centro de estudios, no necesitando invertir mucho tiempo ni dinero para ellas.
Debemos agregar que es propio de la clase media y alta el uso frecuente de Internet en el hogar, así el 74,1\% del sector A y B en el Perú señaló su uso el mes anterior (Marketing Data, 2012). El $83.4 \%$ de los universitarios encuestados señalan que han pasado bien o muy bien el fin de semana. Entre las actividades que más ocupa a la mayoría de jóvenes en su entretenimiento o descanso durante el fin de semana tenemos:

Más de 4 horas: compartir con los amigos 37,3\% o el enamorado $28,5 \%$, así como comunicarse por Internet $27,8 \%$. De l a 4 horas: ver televisión, videos o ir al cine, leer libros, ocupan la mayor parte de su tiempo de descanso.

Vemos asíque los estudios absorben su tiempoyla dedicación a otras actividades se reduce bastante. Por lo que podemos concluir que el consumismo no es algo muy característico en sus vidas, como se podría pensar por su edad y extracción de clase, aunque las horas ocupadas en redes sociales es alta. Los jóvenes universitarios del estudio son conservadores, austeros, responsables y buscan el progreso personal.

Debemostenerpresentequelaprofesionalización aún guarda importancia entre las clases medias y otras como instrumento de ascenso social, pero ha perdido su encanto y la seguridad del status que antes brindaba; pero los jóvenes del 
estudio siguen apostando por la educación como vehículo para esto, posiblemente la experiencia paterna tiene aquí una gran relevancia, en tanto la mayoría tiene padres que están académicamente muy calificados.

Los valores de la cultura "eficientista" son afines con la educación universitaria en la Universidad Peruana Cayetano Heredia, ya que es muy competitiva; de tal manera que los estudiantes siempre están en busca de la eficacia de sus actos para alcanzar logros.

Además del "eficientismo", el concepto de la "individualidad perfecta", también esinteresante.

Este último concepto se refiere a la figura dominante del hombre o mujer joven, profesional, dinámico, innovador, creativo y que es capaz de alcanzar sus metas de manera planificada y que está muy adaptada a la realidad; muy eficiente en el cumplimiento de tareas y objetivos pero con una muy reducida capacidad de hacer sintonía con las propias emociones y deseos. Este último concepto se complementa con el de la "personalidad muy adaptada", la cual implica la transmisión desde pequeños de un ideal acerca de lo que hay que hacer en la vida, transmitiendo un fuerte sentido del trabajo.

El resultado son personas que son muy capaces para hacer ciertas actividades que aseguren una sobre adaptación sin dificultades para el entorno, pero esto implica también el consumo de tranquilizantes, estimulantes, remedios contra las gastritis son cada vez mayores, las enfermedades psicosomáticas y las depresiones todo dentro escenarios exitosos (Guillermo Nugent, 2003). Pero los jóvenes de nuestra investigación, tal vez por su juventud, no se encuentran en esos trances porque nuestra clase media no tiene tal grado de individualidad perfecta que plantea Nugent. Es así que nosotros encontramos que el grado de satisfacción con las amistades es muy alto (84,3\%), le sigue el grado de satisfacción con los estudios $(78,8 \%)$ y el grado de satisfacción con la familia $(74,8 \%)$.
Es de resaltar el hecho de que valoren mucho la amistad y que dada la falta de salud mental que impera en estos tiempos, las respuestas son muy satisfactorias y no denotan mayor descontento; pero es posible que por su juventud, el estrés de las urgencias económicas esté postergado.

\section{Conclusiones}

1. Los jóvenes universitarios del estudio sí perciben la desigualdad en el Perú, ellos consideran que existe poca o ninguna preocupación de las instituciones por la situación de la población indígena en $78.8 \%$ y respecto a la población campesina en $71.5 \%$, vemos que tienen un grado importante de conciencia social. Pero las mujeres tienen una opinión más crítica sobre la desigualdad entre regiones del Perú, ya que las mujeres señalan que ésta es poco satisfactoria, en cambio los varones dicen que ésta es regular.

2. Sobre la igualdad de oportunidades, las mujeres muestran más descontento que los varones, pero no existen diferencias significativas a nivel estadístico, así los varones señalan que esta es regular y según las mujeres que es poco satisfactoria.

3. Sobre la situación económica del Perú, las mujeres tienen una posición más crítica que los varones, existiendo diferencias estadísticas significativas entre varones y mujeres, ya que los varones están más satisfechos que las mujeres, aunque las mujeres tienen una perspectiva más optimista sobre el futuro del país.

4. Los varones muestran menor flexibilidad respecto al grupo y las mujeres muestran mayor colectivismo, en tanto los varones exigen a las demás personas mayor tolerancia, en cambio las mujeres exigen menos al respecto, mostrando mayor flexibilidad al entorno social.

5. El estilo de vida que tienen los jóvenes en la universidad no evidencia un afán de 
ostentación con vestimentas de marca. El consumismo no es algo característico en sus vidas, como se podría pensar por su edad y extracción de clase, aunque las horas ocupadas en redes sociales es alta. Lo que si hemos podido apreciar es la presencia de la cultura "eficientista".

6. La vocación profesional y el elegir determinado centro de estudios, se vincula a un tipo de socialización de los jóvenes dentro de su círculo familiar, amical y dentro de algunos medios de comunicación. El

\section{REFERENCIAS}

Buitron, Ayme y Carbajal, Luz (2013). Percepción de la realidad social del país y la imagen de la universidad de los estudiantes de la Universidad Peruana Cayetano Heredia. En; Percepción de la sociedad e imagen de la universidadopiniones y valoraciones de estudiantes universitarios de Argentina, Bolivia, Brasil, Chile, España, Paraguay y Perú (287). España: TGD.

Buitrón, Ayme y Carbajal, Luz (2010). La percepción de la conservación del medio ambiente de estudiantes de la Universidad Peruana Cayetano Heredia. En: La percepción de la conservación del medio ambiente - opiniones, valoraciones y actitudes de estudiantes universitarios de Argentina, Brasil, Chile, España, Paraguay, Perú y Uruguay (384). España: TGD.

Marketing Data (2012). Niveles socio económicos de Lima Metropolitana. Año 12_223, de Ipsos Apoyo. Sitio web: http://es.scribd.com/doc/150994701/MKT-Data-NSEGran-Lima-2012 significado que adquiere un centro educativo y una profesión, moviliza a los jóvenes a la búsqueda de determinados logros dentro de dicha institución o grupo.

7. Los jóvenes han encontrado mucha motivación en su entorno para el estudio y para enfrentar los retos que encontrarán en ese período de sus vidas.

8. Los jóvenes del estudio son conservadores, austeros, responsables y buscan el progreso personal.
Nugent, Guillermo (2003). Clase media, de la mano invisible a la clase invisible. En: Perú Hoy - la clase media existe? (35). Lima: DESCO.

Palencia Gutiérrez, Esperanza (2006). Individualismo, colectivismo y su relación con la autoestima colectiva de los docentes de enfermería con respecto a los valores educativos de la carrera. España: Tesis doctorales Universidad de Alicante.

Pérez Tarango, G. y García Campos, T. (2010). Individualismo colectivismo y bienestar subjetivo. 1 de Junio 2014, del Departamento de Psicología de la Universidad de Guanajuato. Sitio web: http://www.veranos.ugto. $\mathrm{mx} /$ Memorias/12VeranoCienciaRegionCentro/ documentos/45.pdf. 